This is a postprint version of the following published document:

Peinado, C., Alonso, A., F. Salvador, E., Baselga, J. \& Catalina, F. (2002). Following in situ photoinitiated polymerization of multifunctional acrylic monomers by fluorescence and photocalorimetry simultaneously. Polymer, 43 (19), pp. 5355-5361.

DOI: $10.1016 / \mathrm{S} 0032-3861(02) 00338-5$

(C) Elsevier, 2002

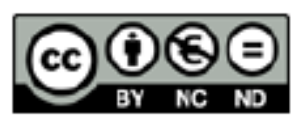

This work is licensed under a Creative Commons Attribution-NonCommercialNoDerivatives 4.0 International License. 


\title{
Following in situ photoinitiated polymerization of multifunctional acrylic monomers by fluorescence and photocalorimetry simultaneously
}

\author{
Carmen Peinado $^{\mathrm{a}, *}$, Asunción Alonso ${ }^{\mathrm{a}}$, Enrique F. Salvador ${ }^{\mathrm{a}}$, Juan Baselga ${ }^{\mathrm{b}, 1}$, Fernando Catalina $^{\mathrm{a}}$ \\ a Instituto de Ciencia y Tecnología de Polímeros, CSIC, Juan de la Cierva 3, 28006 Madrid, Spain \\ ${ }^{\mathrm{b}}$ Instituto Álvaro Alonso Barba, Universidad Carlos III de Madrid, Av. Universidad 30, Leganes, 28911 Madrid, Spain
}

\begin{abstract}
The photoinitiated polymerization of multifunctional (meth)acrylic monomers was simultaneously monitored in real time by fluorescence and differential scanning calorimetry. As the curing proceeds, the fluorescence emission changes due to an increase in the viscosity of the microenvironment. A good correlation between fluorescence intensity and degree of conversion was established by using two different fluorescent probes, each having the same fluorophore, dialkylamino derivatives of 7-nitro-2-oxa-1,3-diazol. One of the probes contains an acrylic moiety which can react with the monomers labelling the formed network. The use of the first moment of fluorescence is presented as a suitable methodology to avoid

experimental fluctuations. Furthermore, the influence of the length of the spacer between the acrylic groups in the polymerization reaction kinetics and fluorescence changes has been also discussed in terms of reaction diffusion controlled termination kinetics and free volume fraction. It was concluded that the fluorescence method is a powerful tool to study the kinetics of photopolymerization of multifunctional monomers.
\end{abstract}

\section{Keywords}

Fluorescence monitoring; Multifunctional acrylates; Photopolymerization

\section{Introduction}

It is well known that the photoinitiated polymerization of multifunctional monomers leads to crosslinked materials, which have found several industrial applications, such as coatings, composites, adhesives, etc. [1]. In the last few decades, the UV-curing technology has expanded due to its advantages: (i) fast curing reactions; (ii) temporal control by switching on or off the light and (iii) spatial control, for example, by using masks to provide an irradiated pattern. From the point of view of energy cost it is more favourable than thermally induced polymerization. Moreover, ecological reasons have promoted the use of solvent-free curable systems. The main efforts have been devoted to an improvement of the photocurable formulations, considering both the development of efficient photoinitiator systems [2, $3]$ and highly reactive monomers and oligomers. However, the aforementioned characteristics of photopolymerization show the need to monitor in real time the process to control

\footnotetext{
* Corresponding author. Tel.: + 34-91-5622900; fax: +34-91-5644853.

E-mail addresses: cpeinado@ictp.csic.es (C. Peinado),

jbaselga@ing.uc3m.es (J. Baselga).

${ }^{1}$ Tel.: +34-91-6249467; fax: +34-91-6249430
}

the physical properties of the final product (quality) and also optimize the conditions of the process.

In spite of the difficulties, due to the heterogeneity and viscosity of the polymerizable systems, different techniques have been applied to study the kinetics of polymerization reaction, even on line [4]. According to the measurement two kind of methodologies have been used: (i) direct, based on the determination of polymer formed or residual monomer and (ii) indirect, that measures the change of a property of the system during polymerization by means of different techniques, such as Fourier transformed infrared spectroscopy, or differential scanning calorimetry (DSC). In the last years, a novel methodology based on fluorescence has been developed for monitoring curing using fluorescent probes incorporated into the curable formulations [5-8]. As polymerization proceeds the rigidity, viscosity and polarity of the microenvironment of the probe change, which in turn, induces a variation in the emitting excited states of the probe, and thus, modifying the fluorescence spectra.

A detailed solvatochromic study of the fluorescence emission of the probes used here was presented in a previous paper [9]. NBD derivatives (dialkylamino derivative of 7-nitro-2-oxa-1,3-diazol) are polarity and viscosity sensitivity probes [10] and we used them to detect the minimum 
concentration of hydrophobic microdomains formation when increasing the concentration of amphiphilic copolymers in aqueous solutions [11]. However, no simple correlation could be established with polarity parameters. For example, the fluorescence maximum of NBD-NEt $\mathrm{N}_{2}$ in $n$-hexane was slightly red-shifted by the addition of ethanol (mole fraction from 0.4 to 1 ). This behaviour was explained as due to specific effects, such as complex formation or dielectric enrichment in the presence of polar solvents.

The information provided by the fluorescent probe needs to be correlated with the properties of the polymer. Many papers deal with the application of the fluorescence method to determine the degree of cure [12-15]. However, mostly fluorescence and the degree of conversion are measured in separated experiments using different techniques where it is difficult to reproduce exactly the experimental conditions. It is well known that photopolymerization rate and degree of conversion depend on experimental conditions (sample thickness, intensity of light,...).

In this paper, we first describe a system designed in our laboratories to accomplish in situ simultaneous measurements of fluorescence spectra and enthalpy curves. Conversion and fluorescence emission from probes has been followed during the photoinitiated polymerization of multifunctional acrylic monomers and their correlation has also been established. The sensor behaviour of a non-reactive fluorescent probe has been compared with that of a reactive acrylic probe, possessing the same fluorophore, dialkylamino derivative of NBD.

\section{Experimental}

\subsection{Materials}

1,6-Hexanediol diacrylate (HDDA), ethylene glycol dimethacrylate (EGDMA), diethylene glycol dimethacrylate (DEGDMA) and poly(ethylene glycol) diacrylates (PEG 258, 575 and 700; where numbers refer to the molecular weights) were purchased from Aldrich. All monomers were used as received.

Fluorescent probes, NBD derivatives, were synthesized as previously described [10]. They were doped into the uncured monomers at small concentration $\left(10^{-5} \mathrm{M}\right)$. The structures of the reactive (NBD-Acr) and non-reactive $\left(\mathrm{NBD}-\mathrm{NEt}_{2}\right)$ probes are shown in Fig. 1. The photoinitiator used in this work was bis(2,4,6-trimethylbenzoyl) phenyl phosphine oxide (TMBAPO, Irgacure 819) provided by Ciba Specialty Chemicals. This photoinitiator acts by fragmentation giving rise to phosphonyl oxide radicals which are very reactive.

\subsection{Optomechanical coupling of a spectrofluorimeter and a photocalorimeter}

A differential scanning calorimeter, Shimadzu model<smiles>CCN(CC)c1ccc([N+](=O)[O-])c2nonc12</smiles>

NBD-NEt $t_{2}$<smiles>C=C([O-])CCCN(C)c1ccc([N+](=O)[O-])c2nonc12</smiles>

\section{NBD-NAcr}

Fig. 1. Chemical structures and abbreviations of the fluorescent probes, NBD-NEt ${ }_{2}$ and NBD-NAcr.

DSC-50, has been modified in-house to carry out photopolymerization and simultaneously, to collect fluorescence in situ. The equipment arrangement is shown in Fig. 2.

The optical assembly of the spectrofluorimeter to the photocalorimeter has been successfully accomplished using a short wave pass dichroic beamsplitter (CVI, SWP-45R480-T400-PW1025-UV) to separate the irradiation light and fluorescence emission. This dichroic beamsplitter shows high transmission at short wavelengths $(\lambda<$ $440 \mathrm{~nm})$ and high reflectivity at longer wavelengths $(\lambda=$ $500-600 \mathrm{~nm})$. Thus, the beamsplitter was placed at $45^{\circ}$ respect to the plane of the cell holders in a modified head of the calorimeter.

In this way, the samples $(40 \mu l)$ were located in the holder of the photocalorimeter. They were then front-face irradiated through an optical fibre with polychromatic light provided by a $400 \mathrm{~W}$ Hg-lamp (Macam-Flexicure). This

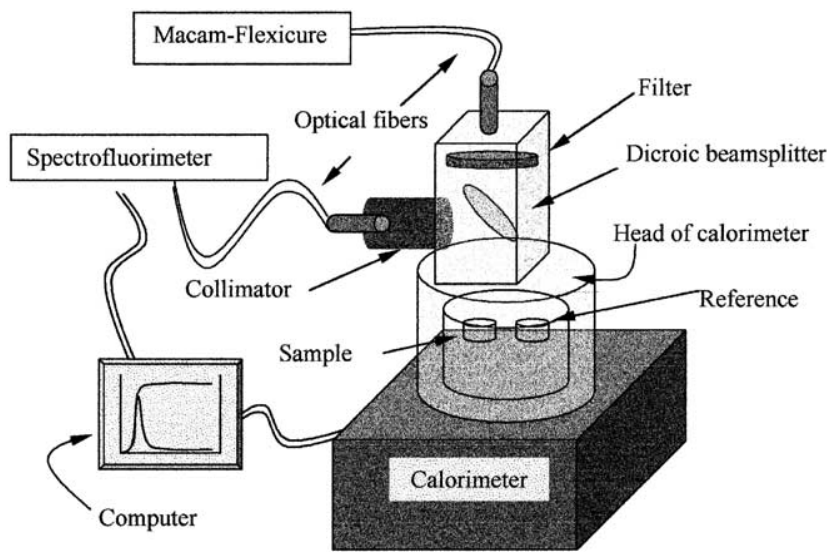

Fig. 2. Scheme of the optical coupling of a spectrofluorimeter and a photocalorimeter. 
light also acts as an excitation source for the fluorescent probes doped into the UV-curing formulations. A cut-off filter (from Hanovia) was used to eliminate wavelengths higher than $430 \mathrm{~nm}$ which interfere with the fluorescence emission spectrum. The spot size was controlled to simultaneously irradiate the sample and the reference holders of the photocalorimeter. Photoinitiator concentration $(0.1 \% \mathrm{w} / \mathrm{w})$ and light intensity $\left(0.6 \mathrm{~mW} \mathrm{~cm}^{-2}\right)$ were kept constant in all the experiments. The samples were irradiated under a nitrogen atmosphere.

Fluorescence is collected with a fibre collimator (Spindler and Hoyer) into an optical fibre, positioned at $90^{\circ}$ respect to the UV-excitation beam and emission is recorded by a Perkin-Elmer spectrofluorimeter LS-50, operated in bioluminescence mode to avoid excitation light from the apparatus. Fluorescence emission is generated from the total polymerization volume allowing better signal-noise ratio.

Heat flow data versus time were recorded isothermally during the polymerization. The polymerization rate (in units of fractional double bond conversion per second) was related to the heat flow monitored by DSC, and the timedependant double bond conversion was calculated by numerical integration of the rate data, taking the values of 56 and $77 \mathrm{~kJ} \mathrm{~mol}^{-1}$ as the theoretical enthalpy for complete conversion for methacrylate and acrylate double bonds, respectively [16].

\section{Results and discussion}

\subsection{Kinetics study of multifunctional (meth)acrylates photopolymerization}

Di(meth)acrylic monomers were photopolymerized in bulk at $40{ }^{\circ} \mathrm{C}$ under a nitrogen atmosphere using as photoinitiator TMBAPO in the presence of a fluorescent probe. The polymerization rate is plotted against conversion in Fig. 3. Previously, it was verified that the effect of probe concentration or probe presence/absence had no influence on the rate of polymerization.

Slight differences in conversion and polymerization rate were observed during the photopolymerization of the same monomer using the different fluorescent probes due to experimental error (differences in sample weight, incident intensity of light, photoinitiator concentration). Moreover, this fact cannot be attributed to the copolymerization of the reactive fluorescent probe, NBD-Acr, which is incorporated into the monomer bulk in a very small concentration. The differences in photophysical behaviour between the reactive and non-reactive fluorescent probes will be pointed out later.

From Fig. 3, an increase of polymerization rate is observed up to reach a maximum and then, the polymerization rate falls to almost zero even though an appreciable amount of monomer exists in the polymerizing mixture.

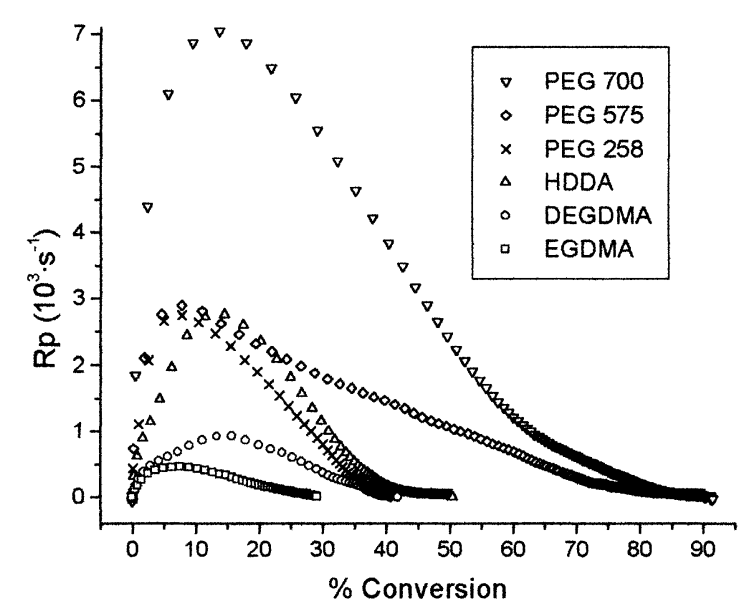

Fig. 3. Plot of polymerization rate versus conversion during the photoinitiated polymerization of the di(meth)acrylates in bulk under nitrogen at $40{ }^{\circ} \mathrm{C}$.

This behaviour corresponds to typical bulk free radical polymerization run at temperatures lower than the glass transition temperature of the polymer. It is well known for multifunctional monomers to reach a conversion lower than $100 \%$. In our experiments the limiting conversion varies from 30 to $80 \%$ depending on the monomer structure.

Table 1 collects the data of the polymerization rate at $5 \%$ conversion $\left(R_{\mathrm{p}}\right)$; the maximum rate of polymerization $\left(R_{\mathrm{p} \text { max }}\right)$, the double bond conversion at the maximum polymerization rate $\left(p_{\max }\right)$ and limiting conversion $\left(p_{\text {lim }}\right)$.

When increasing the length of the chain spacer (ethylene glycol units) between the acrylic moieties the distance between intercrossing points in the formed network also increases. Therefore, the rigidity of the network diminishes, vitrification occurs at higher degrees of conversion and the limiting conversion increases. In this sense, a clear correlation is observed between the limiting degree of conversion and the number of ethylene glycol units between acrylate groups. This fact seems to indicate that the accessibility to the reactive sites, as well as the double bonds, depends on topological factors, at least in the last stages of the photopolymerization reaction. This result is in accordance with reaction diffusion controlled termination kinetics. Mobility through reaction diffusion becomes easier than other forms of diffusion for radicals due to the highly

Table 1

Kinetic data of photopolymerization of difunctional (meth)acrylic monomers in bulk under $\mathrm{N}_{2}$ atmosphere at $40^{\circ} \mathrm{C}$. TMBAPO was used as photoinitiator

\begin{tabular}{llcll} 
Monomer & $R_{\mathrm{p} \max } \times 10^{3}$ & $p_{\max }(\%)$ & $R_{\mathrm{p}} \times 10^{3}$ & $p_{\text {lim }}(\%)$ \\
\hline EGDMA & 0.5 & 9 & 0.4 & 30 \\
DEGDMA & 0.9 & 16 & 0.7 & 42 \\
HDDA & 2.5 & 12 & 1.8 & 51 \\
PEG 258 & 3.0 & 6 & 2.2 & 41 \\
PEG 575 & 4.1 & 12 & 4.0 & 80 \\
PEG 700 & 7.0 & 13 & 5.2 & 92 \\
\hline
\end{tabular}

$R_{\mathrm{p}}$ in $\mathrm{mol} \mathrm{l}^{-1} \mathrm{~s}^{-1} . I_{0}=0.6 \mathrm{~mW} \mathrm{~cm}{ }^{-2}$. 
crosslinked structure formed during the photopolymerization of the multifunctional acrylates $[17,18]$.

Longer spacers between the acrylic double bonds favour their diffusion. In this regard, the maximum values of the polymerization rate $\left(R_{\mathrm{p} \text { max }}\right)$ and $R_{\mathrm{p}}$ increase with the dimensions of the spacer for both acrylic and methacrylic monomers. In the earlier stage of polymerization branches are generated in the growing macroradical, limiting its translational diffusion inside the reactive bulk. Thus, the maximum polymerization rates are reached at low conversions (6-16\%) depending on the monomer structure.

\subsection{Fluorescence monitoring of multifunctional (meth)acrylates photopolymerization}

The photostability of the probe was first checked by UV-absorption monitoring under curing conditions. A constant absorption was fulfilled indicating that photodegradation of the probes does not occur at longer irradiation times than those required for photopolymerization. Both fluorescent probes show maximum emission in the wavelength range $(520-534 \mathrm{~nm})$ where the dicroic beam splitter have the reflection window which makes them adequate for our designed device.

The fluorescence spectra of the probes were measured simultaneously to the heat of polymerization by means of the spectrofluorimeter coupled to the photo-DSC, during the crosslinking of the multifunctional monomers. In this sense it is important to point out that the degree of monomer conversion also increases in the dark, although more slowly. The home-built device allows more confidence and reproducibility to correlate conversion and fluorescence changes during photopolymerization of acrylic monomers doped with fluorescent probes.

Fig. 4 shows the fluorescence spectra of $\mathrm{NBD}_{-\mathrm{NEt}_{2}}$ during the polymerization of HDDA photoinitiated by TMBAPO at $40{ }^{\circ} \mathrm{C}$ under a nitrogen atmosphere. It is important to note that fluorescence is collected at the

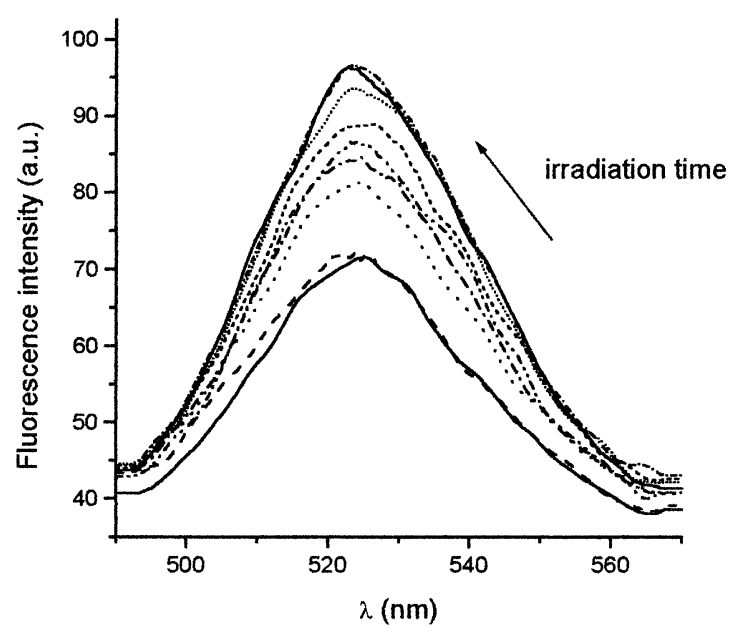

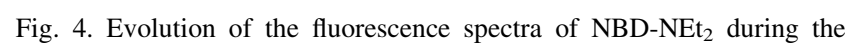
photoinitiated polymerization of HDDA in bulk under nitrogen at $40{ }^{\circ} \mathrm{C}$. polymerization temperature $\left(40{ }^{\circ} \mathrm{C}\right)$, thus, fluorescence changes are related to changes in the free volume fraction during UV-curing [15]. As free volume fraction depends on temperature more reliable correlation can be established here than when fluorescence is collected at room temperature. The fluorescence intensity at the maximum shows good sensitivity during the whole range of conversion of the photopolymerization of the studied monomers. The fluorescence intensity at the initial reaction time was used as an internal standard to calibrate any fluctuation during the curing process and from different experiments.

Fluorescence intensity increases due to the increasing viscosity surrounding the probe during photopolymerization. This behaviour has been interpreted by assuming that the intramolecular charge transfer excited state relaxes to a lower energy twisted intramolecular charge transfer state by rotation about the amino group carbon-nitrogen bond [19]. The radiative deactivation constant is not affected, but a decrease in the non-radiative deactivation constant is related to an increase in the viscosity [10].

Fig. 5 shows the plots of fluorescence intensity versus conversion during the photopolymerization of (a) EGDMA and DEGDMA and (b) the series of PEG, using NBD-NEt 2 as fluorescent probe.

All the data fall on a single smooth curve whose slope is much sharper at the later stages of cure, except in the case of PEG 575 and PEG 700. In other words, the fluorescence emission is a very sensitive monitoring method for cure. Further, the role of the spacer length is present again. The free volume fraction decreases as the distance between reactive acrylic groups does, leading to a decrease in the molecular mobility of the probe. This fact together with viscosity increase leads to a decrease of the non-radiative deactivation rate of the excited states and fluorescence increases. However, the diethylamino group is a small rotor, and its rotation may be more closely related to free volume effects than to macroscopic viscosity which is expected to play a minor role [20]. Fig. 5(a) shows a sharp fluorescence intensity increase at the conversion range from 20 to $28 \%$ with EGDMA; whereas the fluorescence increases smoothly with DEGDMA. The series of poly(ethylene glycol) diacrylates shows a similar behaviour as a result of the different variations in viscosity during the photopolymerization.

Several authors have pointed out that DSC technique monitors the polymerization rate rather than conversion and this method fail for conversion measurements in the case of very slow processes [21]. This fact can explain the broken correlation between fluorescence and DSC conversions at the final stage of polymerization as follows. Polymerization rate decreases as results of vitrification, below the detection limit of DSC. However, diffusion of free monomer is still possible as well as transport of reactive sites via tertiary hydrogen atom abstraction and conversion grows up during irradiation until a topological limit is reached [22]. Therefore photopolymerization continues in the glassy state 

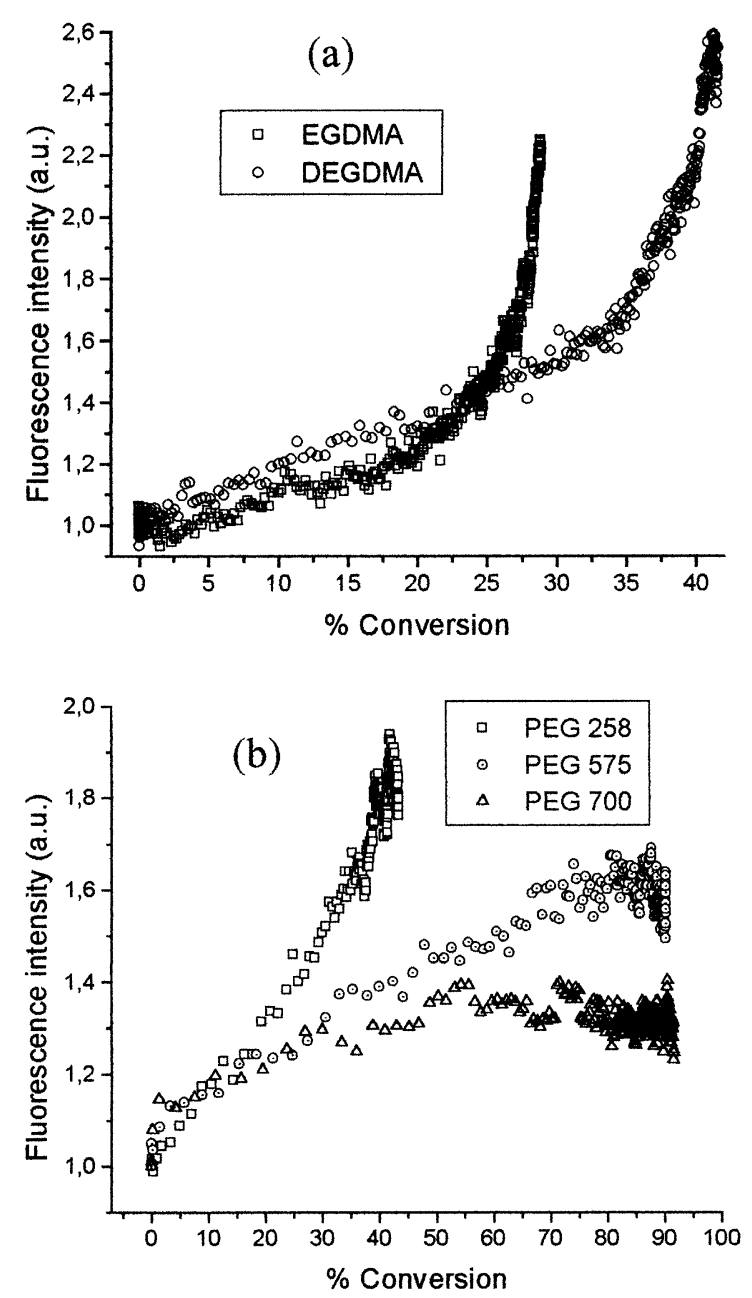

Fig. 5. Plot of the normalized intensity of fluorescence emission at the maximum wavelength versus the degree of conversion of: (a) EGDMA and DEGDMA and (b) the series of PEG diacrylates (with different number of ethylene glycol units between acrylic functionalities). Fluorescent probe: $\mathrm{NBD}-\mathrm{NEt}_{2}$

giving rise to the fluorescence increase as far as free volume fraction changes.

In this work, two fluorescent probes, possessing the same fluorophore, has been used. One of them has a reactive acrylate moiety to be anchored to the formed network during the irradiation. In general, a lower emission intensity is observed for acrylic monomers containing an electrondonating chromophore than that of the saturated analogue. This phenomenon, termed as fluorescence structural selfquenching effect (SSQE) [23], has been used for monitoring the cure reaction in bismaleimide resins [24]. However, SSQE was not observed for NBD-NAcr, due to the ethylene chain spacer between the chromophore and the acrylic double bond, and thus, behaves as an environment-sensitive fluorescent probe. As those fluorescent probes are sensitive to the changes in their microenvironment a different behaviour can be expected depending on their mobility which is restricted as the polymerization proceeds in the case of NBD-NAcr. Fig. 6 shows that the intensity

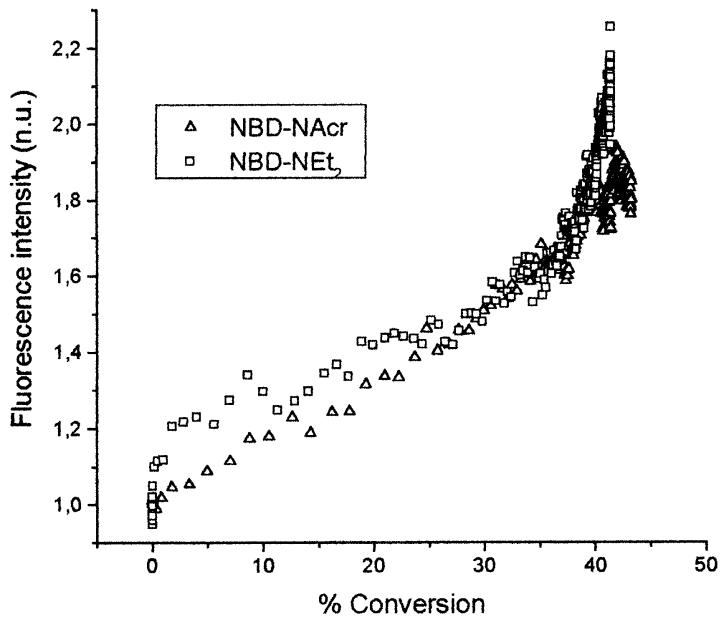

Fig. 6. Comparison of the behaviour of the non-reactive and acrylic probes, NBD-NEt $t_{2}$ and NBD-NAcr, respectively. Plot of the fluorescence intensity as a function of the irradiation time during the photopolymerization of PEG 258.

variations as conversion grows follow the same profile for both probes. No differences are observed between the acrylic and non-reactive probes. This feature can be related to a low reactivity constant of the acrylic double bond with a pendant bulky group of the NBD-NAcr compared to that of the di(meth)acrylic monomers. During the last stage of polymerization a pronounced increase of fluorescence is observed with NBD-NEt 2 , corresponding to post-polymerization processes. This effect is lower in the case of the acrylic probe, pointing out that, probably, the formed network is labelled with the fluorophore at the end of the reaction and the motion restrictions diminish the sensitivity of the label. This is in accordance with our results using a polymeric probe, a copolymer of methyl methacrylate with NBD-NAcr, whose fluorescence emission does not change during the photopolymerization of these monomers. These observations may indicate insufficient separation of the pendent fluorophores and/or restricted mobility of the polymer backbone [25].

The value of the fluorescence intensity at the maximum depends on experimental factors such as incident intensity, probe concentration, etc. Baselga and co-workers [26] have proposed to use a new method based on the determination of the first fluorescent moment to monitor both free radical polymerization and step-growth polymerization. The first moment of emission spectrum [27], $\langle\nu\rangle$, is given by the weighted average wavenumber in Eq. (1):

$\langle\nu\rangle=\sum I_{\mathrm{F}}(\nu) \nu / \sum I_{\mathrm{F}}(\nu)$

In Fig. 7 both fluorescence intensity and $\langle\nu\rangle$ are plotted as a function of conversion for the polymerization of HDDA in the presence of NBD-NEt 2 . The mathematical treatment of the experimental data (emission spectra between 500 and $600 \mathrm{~nm}$ ) for calculation of the first moment has led to a more 


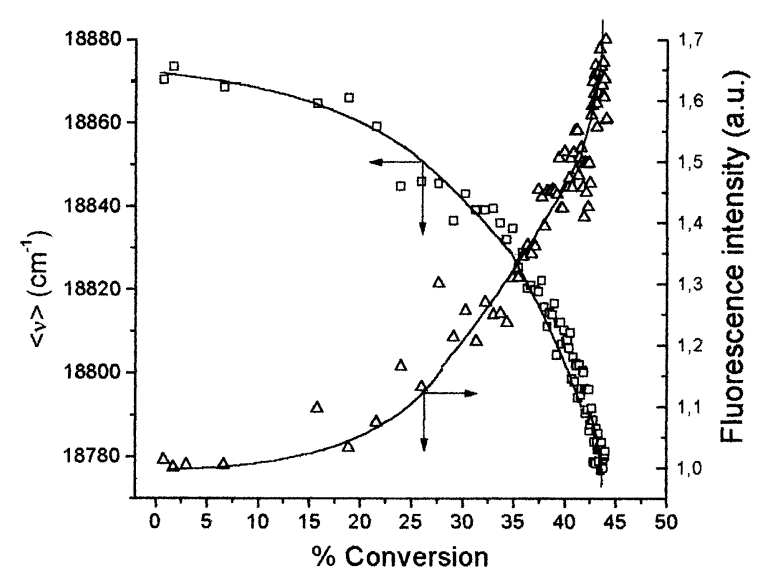

Fig. 7. Comparison between fluorescence intensity and the first moment of the fluorescence emission versus conversion during the photoinitiated polymerization of HDDA in the presence of the probe NBD-NEt 2 .

accurate correlation between fluorescence and degree of conversion with a lower scatter of data. Also, it shows high sensitivity at the range of high conversion which is of great interest from an applied viewpoint. Moreover, the intensity method requires an internal standard or normalization to avoid errors due to experimental fluctuations while the first moment of fluorescence gives absolute measurements.

The origin of the changes in the emission spectra is rather complex. In solvatochromic probes with a strong dipole moment in the excited state the typical behaviour during a curing reaction consists of shifting the average emission energy towards higher energies due to an increasingly inefficient coupling between the excited state dipole and the environment. In the NBD-NEt 2 case, the behaviour is opposite and it seems that as curing proceeds, the probe relaxes around $90 \mathrm{~cm}^{-1}$ through a more stable state. The origin of this relaxation is presently quite unclear. The moderate value of the excited state dipole moment [10] of this probe may influence the capability to be stabilized by its environment by means of electrostatic interactions. Further work is needed to elucidate this behaviour.

\section{Conclusions}

To the best of our knowledge, this is the first report analysing simultaneously fluorescence emission and heat of reaction during the photopolymerization of di(meth)acrylic monomers. Because of post-cure processes and physical ageing alter the structure of the formed network it is of great importance to measure in the same conditions to establish good correlations between fluorescence and degree of conversion.

In conclusion, we have shown that alkylamino derivatives of NBD are sensitive probes for accurately monitoring photopolymerization of multifunctional (meth)acrylic monomers. The effect of the length of the spacer between the acrylic double bonds influence the kinetics of poly- merization and thus, fluorescence response. The microstructural differences in the formed heterogeneous networks are responsible of the changes in the fluorescence spectra. Confirmation of the first moment of fluorescence as a valuable parameter for probe monitoring method has been obtained in this work.

\section{Acknowledgments}

The authors would like to thank the Union European Commission for funding through the BRITE-Euram Project (BE97-4472). Gratitude is also extended to Plan Nacional I + D + I (Ministerio de Ciencia y Tecnología) for financial support (MAT1998-0518-CE and MAT2000-1671). We thank to Dr K. Dietliker, from Ciba Speciality Chemicals, for providing the photoinitiators. The authors are grateful to Valentín Guadaño from Lasing for helpful discussions regarding to the optical coupling between the calorimeter and spectrofluorimeter. J. Baselga wishes to thank CAM (PRICIT Programme).

\section{References}

[1] Fouassier JP. In: Fouassier JP, Rabek JF, editors. Radiation curing in polymer science and technology, vols. 1 and 7. London: Elsevier; 1993. chapter 2.

[2] Catalina F, Peinado C, Blanco M, Alonso A, Allen NS. J Photochem Photobiol A, Chem 2000;131:141.

[3] Peinado C, Alonso A, Catalina F, Schnabel W. Macromol Chem Phys 2000;201:1156.

[4] Kammona O, Chatzi EG, Kiparissides C. JMS: Rev Macromol Chem Phys 1999;C39(1):57.

[5] Jager WF, Volkers AA, Neckers DC. Macromolecules 1995;28:8153.

[6] Wang ZJ, Song JC, Bao R, Neckers DC. J Polym Sci, Polym Phys 1996;34:325.

[7] Yu JW, Sung CSP. J Appl Polym Sci 1997;63:1769.

[8] Strehmel B, Malpert JH, Sarker AM, Neckers DC. Macromolecules 1999;32:7476.

[9] Peinado C, Salvador EF, Baselga J, Catalina F. Macromol Chem Phys 2001;202:1924.

[10] Fery-Forgues S, Fayet JP, Lopez A. J Photochem Photobiol A, Chem 1993;70:229.

[11] Alonso A, Catalina F, Salvador EF, Peinado C. Macromol Chem Phys 2001;202:2293.

[12] Jager WF, Lungu A, Chen DY, Neckers DC. Macromolecules 1997; 30:780.

[13] Serrano B, González-Benito J, Cabanelas JC, Bravo J, Baselga J. J Fluoresc 1997;7:341.

[14] Peinado C, Fernández-Arizpe A, Catalina F, Mateo JL, Bosch P. Macromol Chem Phys 2002;203:336.

[15] Peinado C, Salvador EF, Catalina F, Lozano AE. Polymer 2001;42: 2815.

[16] Odian G. Principles of polymerisation. New York: Wiley; 1991.

[17] Anseth KS, Wang CM, Bowman CN. Macromolecules 1994;27:650.

[18] Mateo JL, Calvo M, Bosch P. J Polym Sci, Polym Chem 2002;40:120.

[19] Rettig W. Appl Phys B 1988;45:145.

[20] Loufty RO, Arnold BA. J Phys Chem 1982;86:4205.

[21] Kloosterboer JG, Litjen GFCM. Polymer 1990;31:95.

[22] Oleinik EF. Adv Polym Sci 1986;80:49. 
[23] Wu SK, Li FM. New trends in photochemistry of polymers. New York: Elsevier; 1985. p. 85.

[24] Zhang X, Du FS, Li ZC, Li FM. Macromol Rapid Commun 2001;22: 983.

[25] Weber SE. Chem Rev 1990;90:1469

[26] Mikes F, González-Benito J, Baselga J. J Macromol Sci, Phys 2001; B40:405.

[27] Leezenberg PB, Frank CW. Macromolecules 1995;28:7407. 